\title{
Autophagy in neuronal development and plasticity
}

4

Angeleen Fleming ${ }^{1,2,3}$ and David C. Rubinsztein ${ }^{1,3^{*}}$

6

$7 \quad{ }^{1}$ Department of Medical Genetics, University of Cambridge, Cambridge Institute for Medical

8 Research, The Keith Peters Building, Cambridge Biomedical Campus, Hills Road,

9 Cambridge, CB2 0XY. United Kingdom.

$10{ }^{2}$ Department of Physiology, Development and Neuroscience, University of Cambridge,

11 Downing Street, Cambridge CB2 3DY, UK.

$12{ }^{3}$ UK Dementia Research Institute, University of Cambridge, Cambridge Institute for Medical

13 Research, The Keith Peters Building, Cambridge Biomedical Campus, Hills Road,

14 Cambridge, CB2 0XY. United Kingdom.

15

16

*Corresponding author: dcr1000@cam.ac.uk [D.C. Rubinsztein]

17

18

19

20 Keywords: autophagy, neuronal stem cell (NSC), neurogenesis, plasticity, memory,

21 psychiatric disease

22

23 
Abstract

Autophagy is a highly conserved intracellular clearance pathway in which cytoplasmic contents are trafficked to the lysosome for degradation. Within neurons, it helps to remove damaged organelles and misfolded or aggregated proteins and has therefore been the subject of intense research in relation to neurodegenerative disease. However, far less is understood about the role of autophagy in other aspects of neuronal physiology. Here we review the literature on the role of autophagy in maintaining neuronal stem cells and in neuronal plasticity in adult life, and we discuss how these contribute to structural and functional deficits observed in a range of human disorders.

\section{Main text}

\section{Autophagy machinery}

Autophagy is a highly conserved catabolic process for clearance of cytoplasmic contents targeted for degradation. In the initial steps of the process, a double-membraned cup-shaped precursor (the phagophore) forms within the cytoplasm. The phagophore expands, surrounding and engulfing substrates as it does so, until the edges fuse and a double-membraned vesicle, the autophagosome, is formed. This is trafficked along microtubules to the part of the cells where lysosomes are concentrated (the microtubule organising centre) to facilitate autophagosome-lysosome fusion, ultimately resulting in the degradation of the autophagosome contents (Figure 1). Much of the core autophagic machinery is controlled by so-called ATG proteins (see Glossary and Box 1). However, many other proteins and processes impact autophagy. Autophagosome formation is induced by diverse signals, including nutrient depletion, and is mediated by many different signalling pathways, including mTORC1 inhibition and AMPK activation [1]. Autophagosome formation involves inputs from other membrane trafficking machineries, including various SNAREs and ESCRT components, and maturation of neuronal autophagosomes may require prior fusion with endosomes [1]. Thus, altered biology of many different cellular systems can impact autophagy.

In addition to bulk degradation of cytoplasmic contents, the recruitment of selective cargoes can be enhanced by so-called autophagy receptors, which typically bind cargoes via ubiquitinated residues and interact with autophagosomes via motifs that bind the core autophagy protein family LC3 (ATG8). Such selective autophagy facilitates degradation of 
organelles like dysfunctional mitochondria (via mitophagy), peroxisomes, and ER, as well as aggregate-prone proteins [2].

Here, we discuss the emerging literature on the role autophagy in the processes of neurogenesis and neuronal plasticity and how compromised autophagy may contribute to structural and functional deficits observed in a range of human disorders (Figure 2). In general, most studies which have examined the roles of autophagy in various physiological settings (including neuronal functions) have used mice or other model organisms with whole body or conditional/selective knockouts of Atg genes. These approaches often need to be viewed with some caution, since some proteins encoded by autophagy genes may have non-autophagic functions [3, 4] (Box 2) or function in related degradation pathways requiring numerous ATG proteins [5,6]. Thus, the interpretation of studies using single knockouts of Atg genes needs to be approached with care, unless corroborating evidence is provided to support the specific role for autophagy in the process being proposed. This is relevant in the CNS, as in many other systems, as autophagy-independent roles of ATG-related proteins impact endocytic and phagocytic processes [5, 6] and stress granule disassembly [7], and likely other pathways that are pertinent to neuronal physiology and pathologies.

\section{Autophagy in neurogenesis}

One area of growing interest is the role of autophagy in the maintenance of neuronal stem cells and the proliferation of neural progenitors. Recent studies have shown that core autophagy genes are expressed in the developing CNS (reviewed in [8]) and knockout studies have demonstrated an essential role for autophagy in neurogenesis in the developing embryo [9].

ATG5 is expressed in neural progenitor cells (NPCs) in the embryonic mouse cortex, and silencing of the gene using electroporation of shRNAs led to decreased neuronal proliferation and abnormal growth and branching of cortical neurons, with a concomitant increase in the cells within the subventricular (SVZ) and ventricular zones [10]. Similarly, in Atg16L1 hypomorph mice, the SVZ was expanded and the cortical plate size was reduced [11]. Evidence suggests that these effects may be mediated by autophagic regulation of $\beta$-catenin levels and Notch1.

EVA1 (also known as transmembrane protein 166, TMEM166) is a lysosome- and ERassociated protein with an established role in autophagy and apoptosis based on in vitro studies 
and is widely expressed in the brain during neurogenesis [12,13]. In conditional knockout mice where EVA1 is absent from Nestin-expressing neuronal stem cells (NSCs), decreased self-renewal and differentiation was observed in the cortex without an increase in apoptosis. These effects were mediated via mTOR activation [13]. In apparent contradiction to these findings, Ambral knockout mice display exencephaly and spina bifida as a consequence of neuronal overgrowth, despite showing clear impairment of autophagy [14]. However, since Ambral also functions as a tumour suppressor gene and its deletion results in increased cell proliferation and increased tumorigenesis [15], it is likely that this accounts for the neuronal defects observed in knockout embryos.

\section{Autophagy and neurodevelopmental disorders}

In support of the role of autophagy in neurogenesis, mutations in two known autophagy genes have been identified that result in microcephaly. A missense (R2637W) mutation in WD repeat and FYVE domain-containing 3 (WDFY3; also known as Autophagy-Linked FYVE or $A L F Y$ ) has been identified as causing human autosomal dominant microcephaly [16]. This scaffolding protein is involved in the selective degradation of ubiquitinated aggregate-prone proteins by autophagy [17] and clearance of mitochondria via mitophagy [18]. In Drosophila, expression of the mutant protein resulted in a 40-60\% reduction in brain volume [16]. In vitro studies have shown that expression of the mutant protein results in increased WNT signalling, likely via a failure to regulate levels of DVL3 (one of the three human dishevelled proteins) through an autophagy-dependent mechanism. In addition, a further 13 mutations in WDFY3 have been found to be associated with mild non-specific neurodevelopmental delay [19]. These result in protein truncating or missense heterozygous mutations. One novel mutation was identified in the PH domain, resulting in microcephaly, whereas the mutations occurring in other domains of the protein were associated with macrocephaly, autism spectrum disorder and attention deficit hyperactivity disorder. In mice harbouring either a nonsense mutation (leading to a stop just before the WD40 domain) in Wdfy3 identified in a forward genetic screen, or those generated by targeted knockout (Wdfy $3^{\text {lac }}$ ), homozygous mutants die at birth [20]. Analysis of embryonic and P0 stages showed cortical thinning and dysplasia but not alterations in autophagic flux, as measured by P62 and LC3II levels [20] (Box 3). However, since Wdfy 3 is an adaptor for selective autophagy, these assays may overlook a role for clearing specific target proteins. Indeed, an investigation into mitophagy in viable, heterozygous 
$W d f y 3^{+/ l a c Z}$ mice revealed an accumulation of defective mitochondria, as well as deficits in mitochondrial transport [18]. These mice display mild cortical abnormalities and, in cultured Purkinje cells, alterations in network complexity and neurite branching were observed [18].

Vici syndrome is multisystem disorder caused by recessive mutations in EPG5. The main neurological feature of the disorder is agenesis of the corpus callosum, and several clinical studies have noted microcephaly in affected individuals (reviewed in [21]). EPG5 is a eukaryote-specific autophagy protein required for autophagosome-lysosome fusion [22]. Absence of EPG5 in CRISPR knockout HeLa cells results in failure of autophagosomelysosome fusion and, as a consequence, blockage of autophagic flux [23]. Since most of the clinical mutations result in truncations, the pathology has been assumed to arise from a lossof-function (reviewed in [21]). Indeed, patient fibroblasts show increased levels of p62/SQSTM1 and LC3-positive puncta (Box 3) and a reduction in LC3-LAMP1 colocalisation, indicative of a build-up of autophagosomes as a result of failure of autophagosome lysosome fusion, a finding supported by further accumulation of these proteins upon pharmacological autophagy induction [24]. However, EPG5-deficient (knockout) mice do not display defects in neurogenesis [25] but adults have reduced numbers of pyramidal cells in layer 5 of the cortex and in the cerebellum, and degenerative features in motor neurons reminiscent of amyotrophic lateral sclerosis [26]. It is possible that the human mutations result in aberrant protein function that is not phenocopied in null mutant model organisms. Indeed, zebrafish epg5-deficient CRISPR/Cas9 models show no overt physical defects or neuronal deficits, despite showing accumulation of non-degradative autophagic vesicles [27].

Interestingly, the clinical neurological features more commonly associated with genetic mutations in autophagy genes are developmental delay, cognitive decline and functional deficits, rather than structural defects in brain development (see Table 1). It is possible that defects in early neurogenesis may underlie these childhood neurological deficits. However, an alternative (but not necessarily exclusive) hypothesis is that developmental delay and cognitive decline may be a consequence of the requirement for autophagy in maintaining neuronal plasticity, as discussed in subsequent sections. In many cases, it remains unclear whether these phenotypes occur as a consequence of defective autophagy, or from non-autophagy functions of the proteins encoded by these genes (Box 2).

\section{Autophagy and adult neural stem cells}


In addition to a role in embryonic neurogenesis, autophagy is also known to play an important role in the differentiation of adult neural stem cells (NSCs). These cells reside within niches primarily at two locations, the sub-ventricular zone (SVZ) of the lateral ventricle wall and subgranular zone (SGZ) of the dentate gyrus. Autophagic flux is low in NSCs in vitro prior to differentiation but increases during early differentiation [28]. Several conditional knockout studies have been performed where Cre is expressed under the control of the GFAP promoter (GFAP-Cre), where the effects on adult neurogenesis have been assessed (reviewed in [29]). Although GFAP is a widely accepted marker of adult NSCs [30], it is important to consider that this promoter has a wider expression pattern during development. Tissue localisation of GFAP-Cre, assessed by crossing to a Cre-sensitive lacZ reporter has demonstrated that Cre is active throughout the CNS at birth [31]. Therefore, analysis of the role of autophagy genes on adult NSCs using GFAP-Cre may be confounded by the loss of gene expression during postnatal development. There are only a few studies where the role of autophagy in adult NSCs has been assessed with temporal control of the genetic ablation or pharmacological intervention. Retroviral Cre injections into dividing NSCs in dentate gyrus of adult Atg $5^{\text {floxfllox }}$ mice reduced autophagic flux and the survival of the progeny of dividing progenitor cells. Surviving cells differentiate into neuronal cells but with delayed neuronal maturation [32].

The Forkhead Box O family of transcription factors (FOXOs) are likely to be key in the regulation of autophagy in NSCs. In mice, embryonic deletion of FOXO1, 3, and 4 results in accelerated depletion of NSCs in adulthood [33-36]. FOXO3 directly binds to and regulates the induction of many autophagy genes in adult neural stem cells [37], and conditional deletion of FOXO 1,3 or 4 in adult NSCs (using GLAST::CreERT2) impairs autophagic flux in developing neurons and results in altered dendritic and spine morphology in adult-generated neurons [33]. Importantly, this study used GLAST-CreERT2 mice (https://www.jax.org/strain/012586) where, in addition to the tissue-specific driver, tamoxifen was used for the temporal activation of Cre, thereby ensuring restriction of the conditional knockout to adult glia and NSCs.

Importantly, as well as their capacity to self-renew and differentiate into neurons, adult NSCs also differentiate into astrocytes and oligodendrocytes. Suppression of autophagy in cultured rat hippocampal NSCs using lentiviral shRNA to knockdown ATG7 or LC3 resulted in fewer astrocytes, and those which formed had abnormal morphology [28]. Similarly, autophagy plays a role in oligodendrocyte and Schwann cell maturation, hence in initial myelination and in remyelination after injury. In mice, oligodendrocyte-specific deletion of Atg5 results in 
lethality at around post-natal day 12, prior to which, animals have fewer oligodendrocyte precursors and reduced myelination [38]. After neuronal injury, myelin debris is cleared by Schwann cells through a form of selective autophagy named myelinophagy [39] and this clearance is delayed in conditional knockout mice where $\operatorname{Atg} 7$ is deleted only in Schwann cells [40].

\section{Autophagy in neuronal plasticity}

In addition to the aforementioned roles of autophagy in maintaining CNS cell populations, an emerging role for autophagy is in the function of the mature nervous system. In the mammalian brain, structural plasticity is essential for the acquisition of knowledge, consolidation of memory, adaptation of behaviour and for repair following injury. There is growing evidence that autophagy plays a role in neuronal plasticity - the ongoing structural reorganisation of neuronal circuits that involves processes like axonal growth, synaptic assembly, and dendritic spine formation and pruning [41-43].

The clearest evidence for this comes from studies of knockouts of core autophagy genes. Pyramidal neurons in conditional knockout mice with neuronal $\operatorname{Atg} 7$ deletion have more dendritic spines than wildtype siblings, and siRNA knockdown of Atg7 in cultured hippocampal neurons demonstrated this to be a consequence of defective spine pruning rather than increased spine formation [44]. In addition, loss of Atg7 in mouse dopaminergic neurons leads to larger axonal profiles, enhanced stimulus-evoked dopamine release and more rapid presynaptic recovery compared to controls, suggesting that autophagy can provide a brake on presynaptic activity by regulating synaptic vesicle turnover [45]. Some of the effects of autophagy on synapses may also be mediated by glia, since loss of microglial autophagy due to conditional Atg7-knockout impairs synaptosome degradation, increases dendritic spines and synaptic markers and alters connectivity [46]. Autophagosomes form in the presynaptic terminal and there is evidence that this biogenesis is controlled locally within the presynaptic region. Gain- or loss-of-function of the synaptic protein Bassoon is sufficient to suppress or enhance autophagy through a direct interaction with Atg5 [47, 48]. Similarly, deletion of the synaptic protein synaptojanin blocks autophagy at the presynaptic terminal [47, 48], a phenotype replicated by mutations in the SAC1 domain of this protein that occur in rare hereditary forms of Parkinson's disease. 
In animal models, these autophagy-associated changes in neuronal plasticity manifest in a range of behavioural phenotypes, such as cognitive deficits [49], anxiety-like behaviours [50], autism-like behaviours [44, 46, 51] and memory deficits [52]. Although there are limited examples of these behavioural consequences, the evidence for memory defects is more compelling. In the mammalian brain, structural plasticity is essential for the consolidation of memory and, in addition to a role in synaptic and dentritic plasticity, there is growing evidence for a role of autophagy in neurotransmitter release and long-term potentiation and depression (LTP and LTD) [42, 43]. The hippocampus is one of the major neuroanatomical areas involved in learning and memory, and autophagy is upregulated in hippocampal neurons during learning and memory consolidation [52]. Knockdown of key autophagy genes (Beclin1, FIP200 and Atg12) in the hippocampus of young mice or exposure to pharmacological autophagy inhibitors reduces performance in novel object recognition and contextual fear conditioning behavioural tests, demonstrating a requirement of autophagy in the formation of novel memories [52]. In addition, the signalling pathways involved in the upstream regulation of autophagy have also been implicated as necessary for the maintenance of neuronal plasticity. Indeed, in mouse models of fragile X syndrome, hyperactivation of mTORC1 leads to decreased autophagy and an associated increase in dendritic spine density, aberrant morphology and exaggerated LTD in hippocampal neurons. These deficits in plasticity contribute to (novel object recognition) memory deficits observed in mouse models of fragile $\mathrm{X}$ syndrome, and both morphological measures of plasticity and behavioural deficits can be rescued by activation of autophagy in such models [49].

Hippocampal autophagy declines with age, and promoting autophagy is sufficient to partly rejuvenate memory in aged animals. Strikingly, injection of plasma from young animals into older mice ameliorates memory in an autophagy-dependent fashion, and these effects can be attributed to the actions of bone-derived osteocalcin, which acts as a hormonal regulator of hippocampal memory [52]. Similarly, in Drosophila, autophagy within the memory centre (mushroom body) protects against age-related expansion of the presynaptic active zones, which is associated with memory impairment [53]. Furthermore, these ageing effects can be ameliorated by inducing autophagy [54].

However, it is important to highlight that upregulation of autophagy may not be a suitable and simple intervention for the treatment of memory deficits and behavioural disorders. In mammalian cell culture experiments, hyperactivation of the positive autophagy regulator, AMPK, leads to an autophagy-dependent loss of pre-and postsynaptic markers and a decline 
in neuronal network function, suggesting that too much autophagy may be deleterious [55]. These experiments tested autophagy dependence using an ULK1 inhibitors, and inhibition of this protein may have some autophagy-independent effects $[3,7]$. Furthermore, it may not be autophagy per se that controls aspects of neuronal plasticity. Recent work demonstrates that the autophagy proteins involved in LC3 lipidation have a non-canonical function in causing microtubule instability which is essential for synapse remodelling (Box 2) [4].

\section{Suggested links with psychiatric diseases}

Given the role of autophagy in neurogenesis and plasticity, as discussed above, it is perhaps not surprising that autophagy defects have been proposed to contribute to human disorders like depression, bipolar disorder and schizophrenia. A number of studies have correlated antidepressant actions of drugs in mice with their abilities to induce autophagy. Diverse drugs that lower IP3 levels, such as valproate, lithium and carbamazepine, have mood-stabilising properties in humans [56], and induce autophagy via the same IP3-lowering mechanism [57]. However, it is important to consider that these drugs also have other activities. Other antidepressants, like fluoxetine and amitriptyline, also induce autophagy via a mechanism that appears to be dependent on the accumulation of sphingomyelin in lysosomes and Golgi membranes and ceramide in the endoplasmic reticulum. Interestingly, inhibition of autophagy using the Beclin 1 (ATG6 protein) inhibitor, spautin, inhibited the benefits of amitryptiline and fluoxetine on neurogenesis, neuronal maturation and behaviour in stressed mice [58]. Other drugs that induce autophagy, like trehalose [59] and rapamycin [60], also have antidepressantlike properties in mice. These studies suggest that the antidepressant effects of some compounds may be, at least in part, autophagy-dependent.

Autophagy appears to be inhibited in an unpredictable chronic mild stress-induced depressive mouse model, and both autophagy and depressive-like behaviour are rescued by rosiglitazone [61]. Interestingly, the antidepressant fluoxetine induces autophagic flux and mitophagy in primary astrocytes from a chronic mild stress-induced mouse model [62]. Autophagy in microglia may also be important, since knockout of the autophagy protein Atg5 in these cells increased inflammation, reduced BDNF expression and contributed to chronic unpredicted mild stress depression-like behaviour in mice [63]. Andrographolide, a natural product, also induces autophagy along with anti-inflammatory effects and improves a range of behavioural performances in a chronic unpredictable mild stress mouse model of depression [64]. 
In many of these studies it is difficult to know whether the effects are directly due to altered autophagy or whether the changes in autophagy are correlational. While serum levels of the autophagy mediator Beclin 1 appear to be higher in responders to selective serotonin reuptake inhibitors, the study was small and needs replication in a larger cohort [65]. Furthermore, while many studies show correlations supporting the idea that autophagy induction may have antidepressant effects, the lysosomal inhibitor bafilomcyin A1, which blocks autophagic flux, also has such properties in rats exposed to chronic unpredictable mild stress [66].

While there are a number of studies that make links between autophagy and depression, the literature related to schizophrenia is smaller. The expression of various autophagy genes has been reported to be decreased in cortical brain areas affected in schizophrenia $[67,68]$ and in hippocampi of schizophrenic patients post-mortem [69]. In addition, the Disrupted-inSchizophrenia 1 (DISC1) protein, which is implicated in psychiatric disorders, appears to act as a mitophagy receptor [70]. Sequence variants in ULK1 have been associated with schizophrenia [71] as well, and mice hemizygous for the Ulk1 homologue, Ulk2, have decreased cell surface GABA receptor levels, which may be relevant to increased neuronal excitability seen in schizophrenia [72].

\section{Concluding Remarks}

Experimental and clinical data suggest that defects in autophagy cause both structural and functional abnormalities in the brain. There is growing evidence that these may contribute to neurobehavioural changes, memory deficits and various psychiatric conditions, although further studies are required before one can make a conclusive case (see Outstanding Questions). One limitation of many experimental studies is that the contribution of autophagy is typically studied by knockout/knockdown of core autophagy genes and is therefore likely to cause a severe block in the pathway, which is not representative of physiological conditions where ageand disease-related changes result in smaller deficits. Another common limitation is that most studies tend to focus on a narrow range of biological features (e.g. dendrite pruning) and often fail to take into account that these changes in plasticity occur in the context of alterations in proteostasis. For example, to examine the role of autophagy in dendrite pruning, one might study this phenomenon in models where core autophagy genes are knocked out. However, in this scenario, one cannot determine whether the effects seen in the distal dendrite are the result 

of a direct role for autophagy in pruning or the consequence of accumulation of autophagy substrates elsewhere in the neuron and/or non-specific toxicity caused by autophagy blockade.

312 A further limitation of studying human mutations in cell or animal models is that gene function

313 is typically studied by knockout/knockdown or by over-expression of the mutant form of the 314 protein. However, human clinical mutations are more commonly point mutations and may 315 result in truncations with reduced or altered function rather than complete loss-of-function. In 316 addition, one must consider that the presence of a mutant allele may result in gain-of-function 317 or aberrant function of the mutated protein in addition to loss of the wild-type protein. 318 Furthermore, the multiple variants contributing to complex diseases like depression or schizophrenia in any one individual may often be non-coding, and their biological effects in 320 isolation and in combination are generally poorly understood. The growing use of 321 CRISPR/Cas9 editing technologies offers the opportunity to develop more clinically-relevant 322 models. In addition, patient fibroblasts and patient-derived iPSCs offer the potential to directly study the consequences of clinical mutations in patient cells, with their complex genetic makeup, in vitro. Another possible route may be to transplant human iPSC-derived neurons or glia into rodent models.

326 A major challenge will be to find ways to bridge the gaps between mouse models and human 327 diseases and to dissect apart the role of autophagy in distinct processes such as dendritic pruning in the context of a neuron where autophagy is globally perturbed. The further development of tools to manipulate autophagy at the subcellular level will be essential to address these challenges. 
333 Acknowledgements

334 We are grateful for funding from the UK Dementia Research Institute (funded by the Medical 335 Research Council UK (MRC), Alzheimer's Research UK and the Alzheimer's Society), Roger 336 de Spoelberch Foundation, Alzheimer's Research UK, Cambridge Centre for Parkinson-Plus, 337 and National Institute for Health Research Cambridge Biomedical Research Centre. The views 338 expressed are those of the author(s) and not necessarily those of the NHS, the NIHR or the 339 Department of Health and Social Care. Figures created with BioRender (Academic licence). 340 
1. Bento, C.F. et al. (2016) Mammalian Autophagy: How Does It Work? Annu Rev Biochem 85, 685713.

2. Stavoe, A.K.H. and Holzbaur, E.L.F. (2019) Autophagy in Neurons. Annu Rev Cell Dev Biol 35, 477500.

3. Joo, J.H. et al. (2016) The Noncanonical Role of ULK/ATG1 in ER-to-Golgi Trafficking Is Essential for Cellular Homeostasis. Mol Cell 62 (4), 491-506.

4. Negrete-Hurtado, A. et al. (2020) Autophagy lipidation machinery regulates axonal microtubule dynamics but is dispensable for survival of mammalian neurons. Nat Commun 11 (1), 1535.

5. Heckmann, B.L. et al. (2019) LC3-Associated Endocytosis Facilitates beta-Amyloid Clearance and Mitigates Neurodegeneration in Murine Alzheimer's Disease. Cell 178 (3), 536-551 e14.

6. Cunha, L.D. et al. (2018) LC3-Associated Phagocytosis in Myeloid Cells Promotes Tumor Immune Tolerance. Cell 175 (2), 429-441 e16.

7. Wang, B. et al. (2019) ULK1 and ULK2 Regulate Stress Granule Disassembly Through Phosphorylation and Activation of VCP/p97. Mol Cell 74 (4), 742-757 e8.

8. Wu, X. et al. (2013) Autophagy and mammalian development. Biochem Soc Trans 41 (6), 1489-94. 9. Kuma, A. et al. (2017) Autophagy-monitoring and autophagy-deficient mice. Autophagy 13 (10), 1619-1628.

10. Lv, X. et al. (2014) The crucial role of Atg5 in cortical neurogenesis during early brain development. Sci Rep 4, 6010.

11. Wu, X. et al. (2016) Autophagy regulates Notch degradation and modulates stem cell development and neurogenesis. Nat Commun 7, 10533.

12. Wang, L. et al. (2007) TMEM166, a novel transmembrane protein, regulates cell autophagy and apoptosis. Apoptosis 12 (8), 1489-502.

13. Li, M. et al. (2016) EVA1A/TMEM166 Regulates Embryonic Neurogenesis by Autophagy. Stem Cell Reports 6 (3), 396-410.

14. Fimia, G.M. et al. (2007) Ambra1 regulates autophagy and development of the nervous system. Nature 447 (7148), 1121-5.

15. Cianfanelli, V. et al. (2015) AMBRA1 links autophagy to cell proliferation and tumorigenesis by promoting c-Myc dephosphorylation and degradation. Nat Cell Biol 17 (1), 20-30.

16. Kadir, R. et al. (2016) ALFY-Controlled DVL3 Autophagy Regulates Wnt Signaling, Determining Human Brain Size. PLoS Genet 12 (3), e1005919.

17. Filimonenko, M. et al. (2010) The selective macroautophagic degradation of aggregated proteins requires the PI3P-binding protein Alfy. Mol Cell 38 (2), 265-79.

18. Napoli, E. et al. (2018) Beyond autophagy: a novel role for autism-linked Wdfy3 in brain mitophagy. Sci Rep 8 (1), 11348.

19. Le Duc, D. et al. (2019) Pathogenic WDFY3 variants cause neurodevelopmental disorders and opposing effects on brain size. Brain 142 (9), 2617-2630.

20. Orosco, L.A. et al. (2014) Loss of Wdfy3 in mice alters cerebral cortical neurogenesis reflecting aspects of the autism pathology. Nat Commun 5, 4692.

21. Ebrahimi-Fakhari, D. et al. (2016) Congenital disorders of autophagy: an emerging novel class of inborn errors of neuro-metabolism. Brain 139 (Pt 2), 317-37.

22. Wang, Z. et al. (2016) The Vici Syndrome Protein EPG5 Is a Rab7 Effector that Determines the Fusion Specificity of Autophagosomes with Late Endosomes/Lysosomes. Mol Cell 63 (5), 781-95. 23. Hori, I. et al. (2017) Defects in autophagosome-lysosome fusion underlie Vici syndrome, a neurodevelopmental disorder with multisystem involvement. Sci Rep 7 (1), 3552.

24. Cullup, T. et al. (2013) Recessive mutations in EPG5 cause Vici syndrome, a multisystem disorder with defective autophagy. Nat Genet 45 (1), 83-7.

25. Miao, G. et al. (2016) Mice deficient in the Vici syndrome gene Epg5 exhibit features of retinitis pigmentosa. Autophagy 12 (12), 2263-2270. 
26. Zhao, H. et al. (2013) Mice deficient in Epg5 exhibit selective neuronal vulnerability to degeneration. J Cell Biol 200 (6), 731-41.

27. Meneghetti, G. et al. (2019) The epg5 knockout zebrafish line: a model to study Vici syndrome. Autophagy 15 (8), 1438-1454.

28. Ha, S. et al. (2019) Autophagy Mediates Astrogenesis in Adult Hippocampal Neural Stem Cells. Exp Neurobiol 28 (2), 229-246.

29. Casares-Crespo, L. et al. (2018) On the Role of Basal Autophagy in Adult Neural Stem Cells and Neurogenesis. Front Cell Neurosci 12, 339.

30. Morshead, C.M. et al. (2003) The ablation of glial fibrillary acidic protein-positive cells from the adult central nervous system results in the loss of forebrain neural stem cells but not retinal stem cells. Eur J Neurosci 18 (1), 76-84.

31. Zhuo, L. et al. (2001) hGFAP-cre transgenic mice for manipulation of glial and neuronal function in vivo. Genesis 31 (2), 85-94.

32. Xi, Y. et al. (2016) Knockout of Atg5 delays the maturation and reduces the survival of adultgenerated neurons in the hippocampus. Cell Death Dis 7, e2127.

33. Schaffner, I. et al. (2018) FoxO Function Is Essential for Maintenance of Autophagic Flux and Neuronal Morphogenesis in Adult Neurogenesis. Neuron 99 (6), 1188-1203 e6.

34. Paik, J.H. et al. (2009) FoxOs cooperatively regulate diverse pathways governing neural stem cell homeostasis. Cell Stem Cell 5 (5), 540-53.

35. Renault, V.M. et al. (2009) FoxO3 regulates neural stem cell homeostasis. Cell Stem Cell 5 (5), 527-39.

36. Yeo, H. et al. (2013) FoxO3 coordinates metabolic pathways to maintain redox balance in neural stem cells. EMBO J 32 (19), 2589-602.

37. Audesse, A.J. et al. (2019) FOXO3 directly regulates an autophagy network to functionally regulate proteostasis in adult neural stem cells. PLoS Genet 15 (4), e1008097.

38. Bankston, A.N. et al. (2019) Autophagy is essential for oligodendrocyte differentiation, survival, and proper myelination. Glia 67 (9), 1745-1759.

39. Gomez-Sanchez, J.A. et al. (2015) Schwann cell autophagy, myelinophagy, initiates myelin clearance from injured nerves. J Cell Biol 210 (1), 153-68.

40. Jang, S.Y. et al. (2016) Autophagic myelin destruction by Schwann cells during Wallerian degeneration and segmental demyelination. Glia 64 (5), 730-42.

41. Lieberman, O.J. and Sulzer, D. (2019) The Synaptic Autophagy Cycle. J Mol Biol.

42. Kulkarni, V.V. and Maday, S. (2018) Compartment-specific dynamics and functions of autophagy in neurons. Dev Neurobiol 78 (3), 298-310.

43. Stavoe, A.K.H. and Holzbaur, E.L.F. (2019) Axonal autophagy: Mini-review for autophagy in the CNS. Neurosci Lett 697, 17-23.

44. Tang, G. et al. (2014) Loss of mTOR-dependent macroautophagy causes autistic-like synaptic pruning deficits. Neuron 83 (5), 1131-43.

45. Hernandez, D. et al. (2012) Regulation of presynaptic neurotransmission by macroautophagy. Neuron 74 (2), 277-84.

46. Kim, H.J. et al. (2017) Deficient autophagy in microglia impairs synaptic pruning and causes social behavioral defects. Mol Psychiatry 22 (11), 1576-1584.

47. Vanhauwaert, R. et al. (2017) The SAC1 domain in synaptojanin is required for autophagosome maturation at presynaptic terminals. EMBO J 36 (10), 1392-1411.

48. Okerlund, N.D. et al. (2017) Bassoon Controls Presynaptic Autophagy through Atg5. Neuron 93 (4), 897-913 e7.

49. Yan, J. et al. (2018) Activation of autophagy rescues synaptic and cognitive deficits in fragile $X$ mice. Proc Natl Acad Sci U S A 115 (41), E9707-E9716.

50. Xiao, X. et al. (2018) Nicotine alleviates chronic stress-induced anxiety and depressive-like behavior and hippocampal neuropathology via regulating autophagy signaling. Neurochem Int 114, 58-70. 
51. Zhu, J.W. et al. (2019) Absence of TRIM32 Leads to Reduced GABAergic Interneuron Generation and Autism-like Behaviors in Mice via Suppressing mTOR Signaling. Cereb Cortex. 52. Glatigny, M. et al. (2019) Autophagy Is Required for Memory Formation and Reverses Age53. Bhukel, A. et al. (2019) Autophagy within the mushroom body protects from synapse aging in a non-cell autonomous manner. Nat Commun 10 (1), 1318.

54. Gupta, V.K. et al. (2016) Spermidine Suppresses Age-Associated Memory Impairment by Preventing Adverse Increase of Presynaptic Active Zone Size and Release. PLoS Biol 14 (9), e1002563. 55. Domise, M. et al. (2019) Neuronal AMP-activated protein kinase hyper-activation induces synaptic loss by an autophagy-mediated process. Cell Death Dis 10 (3), 221.

56. Williams, R.S. et al. (2002) A common mechanism of action for three mood-stabilizing drugs. Nature 417 (6886), 292-5.

57. Sarkar, S. et al. (2005) Lithium induces autophagy by inhibiting inositol monophosphatase. J Cell Biol 170 (7), 1101-11.

58. Gulbins, A. et al. (2018) Antidepressants act by inducing autophagy controlled by sphingomyelinceramide. Mol Psychiatry 23 (12), 2324-2346.

59. Kara, N.Z. et al. (2013) Trehalose induced antidepressant-like effects and autophagy enhancement in mice. Psychopharmacology (Berl) 229 (2), 367-75.

60. Kara, N.Z. et al. (2018) Mood-stabilizing effects of rapamycin and its analog temsirolimus: relevance to autophagy. Behav Pharmacol 29 (4), 379-384.

61. Zhao, Z. et al. (2017) Rosiglitazone Exerts an Anti-depressive Effect in Unpredictable Chronic Mild-Stress-Induced Depressive Mice by Maintaining Essential Neuron Autophagy and Inhibiting Excessive Astrocytic Apoptosis. Front Mol Neurosci 10, 293.

62. Shu, X. et al. (2019) The effect of fluoxetine on astrocyte autophagy flux and injured mitochondria clearance in a mouse model of depression. Cell Death Dis 10 (8), 577.

63. Tan, X. et al. (2018) Inhibition of Autophagy in Microglia Alters Depressive-Like Behavior via BDNF Pathway in Postpartum Depression. Front Psychiatry 9, 434.

64. Geng, J. et al. (2019) Andrographolide triggers autophagy-mediated inflammation inhibition and attenuates chronic unpredictable mild stress (CUMS)-induced depressive-like behavior in mice. Toxicol Appl Pharmacol 379, 114688.

65. He, S. et al. (2019) Baseline Serum Levels of Beclin-1, but Not Inflammatory Factors, May Predict Antidepressant Treatment Response in Chinese Han Patients With MDD: A Preliminary Study. Front Psychiatry 10, 378.

66. Wang, Z. et al. (2018) Bafilomycin A1 alleviates depressionlike symptoms in chronic unpredictable mild stress rats. Mol Med Rep 18 (5), 4587-4594.

67. Barnes, M.R. et al. (2011) Transcription and pathway analysis of the superior temporal cortex and anterior prefrontal cortex in schizophrenia. J Neurosci Res 89 (8), 1218-27.

68 . Horesh, Y. et al. (2011) Gene expression signature is shared by patients with Alzheimer's disease and schizophrenia at the superior temporal gyrus. Eur J Neurol 18 (3), 410-24.

69. Merenlender-Wagner, A. et al. (2015) Autophagy has a key role in the pathophysiology of schizophrenia. Mol Psychiatry 20 (1), 126-32.

70. Wang, Z.T. et al. (2019) Disrupted-in-schizophrenia-1 protects synaptic plasticity in a transgenic mouse model of Alzheimer's disease as a mitophagy receptor. Aging Cell 18 (1), e12860.

71. Al Eissa, M.M. et al. (2018) Exome sequence analysis and follow up genotyping implicates rare ULK1 variants to be involved in susceptibility to schizophrenia. Ann Hum Genet 82 (2), 88-92. 72. Sumitomo, A. et al. (2018) Ulk2 controls cortical excitatory-inhibitory balance via autophagic regulation of p62 and GABAA receptor trafficking in pyramidal neurons. Hum Mol Genet 27 (18), 3165-3176.

73. Kuma, A. et al. (2004) The role of autophagy during the early neonatal starvation period. Nature 432 (7020), 1032-6. 
74. Hara, T. et al. (2006) Suppression of basal autophagy in neural cells causes neurodegenerative disease in mice. Nature 441 (7095), 885-9.

75. Zhang, J. et al. (2016) A Founder Mutation in VPS11 Causes an Autosomal Recessive Leukoencephalopathy Linked to Autophagic Defects. PLoS Genet 12 (4), e1005848. 76. Zhao, Y.G. et al. (2015) The autophagy gene Wdr45/Wipi4 regulates learning and memory function and axonal homeostasis. Autophagy 11 (6), 881-90.

77. Ji, C. et al. (2019) Role of Wdr45b in maintaining neural autophagy and cognitive function. Autophagy, 1-11.

78. Ohno, M. et al. (2009) Nardilysin regulates axonal maturation and myelination in the central and peripheral nervous system. Nat Neurosci 12 (12), 1506-13.

79. Akizu, N. et al. (2015) Biallelic mutations in SNX14 cause a syndromic form of cerebellar atrophy and lysosome-autophagosome dysfunction. Nat Genet 47 (5), 528-34.

80. Branchu, J. et al. (2017) Loss of spatacsin function alters lysosomal lipid clearance leading to upper and lower motor neuron degeneration. Neurobiol Dis 102, 21-37.

81. Khundadze, M. et al. (2013) A hereditary spastic paraplegia mouse model supports a role of ZFYVE26/SPASTIZIN for the endolysosomal system. PLoS Genet 9 (12), e1003988.

82. Sugimoto, R. et al. (2010) Enhanced neointimal hyperplasia and carotid artery remodelling in sequestosome 1 deficient mice. J Cell Mol Med 14 (6B), 1546-54.

83. Harada, H. et al. (2013) Deficiency of p62/Sequestosome 1 causes hyperphagia due to leptin resistance in the brain. J Neurosci 33 (37), 14767-77.

84. Kimura, T. et al. (2017) Cellular and molecular mechanism for secretory autophagy. Autophagy 13 (6), 1084-1085.

85. Iula, L. et al. (2018) Autophagy Mediates Interleukin-1ß Secretion in Human Neutrophils. Front Immunol 9, 269.

86. Gerstenmaier, L. et al. (2015) The autophagic machinery ensures nonlytic transmission of mycobacteria. Proc Natl Acad Sci U S A 112 (7), E687-92. 
Table 1: Neurodevelopmental defects associated with mutations in genes regulating autophagy

\begin{tabular}{|c|c|c|c|c|}
\hline $\begin{array}{l}\text { Disease name } \\
\text { (OMIM identifier) }\end{array}$ & $\begin{array}{l}\text { Gene } \\
\text { symbol }\end{array}$ & Clinical features & Role in autophagy & Phenotype in animal models \\
\hline $\begin{array}{l}\text { Autosomal recessive } \\
\text { spinocerebellar ataxia } \\
25 ; \text { SCAR25 } \\
\text { (\# 617584) }\end{array}$ & ATG5 & $\begin{array}{l}\text { Delayed psychomotor development, truncal ataxia, } \\
\text { dysmetria, nystagmus, low IQ, cerebellar hypoplasia }\end{array}$ & $\begin{array}{l}\text { Core autophagy gene. As part of the ATG5-12 } \\
\text { conjugate, it is essential in the processing to } \\
\text { form LC3II during autophagosome formation }\end{array}$ & $\begin{array}{l}\text { Atg5 null mice die within } 1 \text { day of birth [73] } \\
\text { whereas conditional knockout in neural } \\
\text { tissue results in progressive } \\
\text { neurodegeneration with motor deficits [74] }\end{array}$ \\
\hline $\begin{array}{l}\text { Vici syndrome; VICIS } \\
\text { (\# 242840) }\end{array}$ & EPG5 & $\begin{array}{l}\text { Multisystem disorder - agenesis of the corpus callosum, } \\
\text { oculocutaneous hypopigmentation, bilateral cataract, } \\
\text { cleft lip and palate, repeated infections suggestive of an } \\
\text { immunodeficiency, cardiomyopathy, postnatal growth } \\
\text { retardation, microcephaly, and profound developmental } \\
\text { delay }\end{array}$ & $\begin{array}{l}\text { Eukaryote-specific autophagy gene required for } \\
\text { autophagosome-lysosome fusion }\end{array}$ & $\begin{array}{l}\text { EPG5-deficient adult mice have fewer } \\
\text { pyramidal neurons in layer } 5 \text { cortex and } \\
\text { cerebellum and develop progressive ALS } \\
\text { phenotype [26]. Zebrafish epg } 5 \text { CRISPR/Cas9 } \\
\text { models show no overt morphological defects } \\
\text { or neuronal deficits [27] }\end{array}$ \\
\hline $\begin{array}{l}\text { Autosomal dominant } \\
\text { primary microcephaly } \\
18, \mathrm{MCPH} 18 \\
(\# 617520)\end{array}$ & WDFY3 & $\begin{array}{l}\text { Microcephaly with mild to moderate intellectual } \\
\text { disability }\end{array}$ & $\begin{array}{l}\text { Scaffolding protein involved in the selective } \\
\text { degradation of ubiquitinated protein aggregates } \\
\text { by autophagy }\end{array}$ & $\begin{array}{l}\text { Homozygous mice with null mutations die at } \\
\text { birth; embryos and PO stages show cortical } \\
\text { thinning and dysplasia [20] }\end{array}$ \\
\hline $\begin{array}{l}\text { Hypomyelinating } \\
\text { leukodystrophy, 12; } \\
\text { HLD12 (\# 616683) }\end{array}$ & VPS11 & $\begin{array}{l}\text { Severely delayed or lack of psychomotor development, } \\
\text { acquired microcephaly, lack of speech, and often lack of } \\
\text { spontaneous movement due to hypotonia and spasticity }\end{array}$ & $\begin{array}{l}\text { Required for the fusion of endosomes and } \\
\text { autophagosomes with lysosomes }\end{array}$ & $\begin{array}{l}\text { zebrafish vps } 11 \text { mutants have apoptotic cell } \\
\text { death in the midbrain and hindbrain and } \\
\text { reduced myelination throughout CNS [75] }\end{array}$ \\
\hline $\begin{array}{l}\text { Neurodegeneration } \\
\text { with brain iron } \\
\text { accumulation 5; } \\
\text { NBIA5* (\# 300894) }\end{array}$ & $\begin{array}{l}\text { WDR45/ } \\
\text { WIPI4 }\end{array}$ & $\begin{array}{l}\text { Biphasic: global developmental delay in early childhood } \\
\text { that is essentially static. Progressive dystonia, } \\
\text { parkinsonism and extrapyramidal signs and dementia } \\
\text { develop in young adulthood }\end{array}$ & $\begin{array}{l}\text { Involved in autophagosome biogenesis } \\
\text { downstream of WIPI2 and regulating the size of } \\
\text { autophagosomes }\end{array}$ & $\begin{array}{l}\text { Wdr45 knockout mice have poor motor } \\
\text { coordination and learning and memory } \\
\text { deficits [76] }\end{array}$ \\
\hline $\begin{array}{l}\text { Neurodevelopmental } \\
\text { disorder with spastic } \\
\text { quadriplegia and } \\
\text { brain abnormalities } \\
\text { with or without } \\
\text { seizures; NEDSBAS } \\
\text { (\# 617977) }\end{array}$ & $\begin{array}{l}\text { WDR45B/ } \\
\text { WIPI3 }\end{array}$ & $\begin{array}{l}\text { Global developmental delay, intellectual disability and } \\
\text { microcephaly }\end{array}$ & $\begin{array}{l}\text { Involved in the control of autophagy upstream of } \\
\text { Ptdlns3P and with WDR45 in regulating } \\
\text { autophagosome size. Also associates with TSC } \\
\text { complex at lysosomes, regulating mTOR }\end{array}$ & $\begin{array}{l}\text { Mice deficient in Wdr45b exhibit motor } \\
\text { deficits and learning and memory defects } \\
\text { [77] }\end{array}$ \\
\hline $\begin{array}{l}\text { NARDILYSIN; NRD1 } \\
(* 602651)\end{array}$ & NRD1 & $\begin{array}{l}\text { Developmental delay, progressive cortical and cerebellar } \\
\text { atrophy, motor impairment, hypotonia, ataxia, absent } \\
\text { speech, seizures, optic atrophy, dysphagia, and } \\
\text { microcephaly }\end{array}$ & $\begin{array}{l}\text { Nrd1 is a mitochondrial co-chaperone for alpha- } \\
\text { ketoglutarate dehydrogenase. Loss of function } \\
\text { results in activation of mTORC1 and a } \\
\text { subsequent reduction in autophagy }\end{array}$ & $\begin{array}{l}\text { Nrd1-null mice have prenatal growth defects } \\
\text { and neonatal lethality. Surviving animals } \\
\text { display slowly progressive } \\
\text { neurodegeneration with impaired motor } \\
\text { activities and cognitive deficits. Mutant mice } \\
\text { have small brains and a thin cerebral cortex } \\
\text { with reduced myelination in CNS and PNS } \\
\text { [78] }\end{array}$ \\
\hline
\end{tabular}




\begin{tabular}{|c|c|c|c|c|}
\hline $\begin{array}{l}\text { Autosomal recessive } \\
\text { spastic paraplegia 49; } \\
\text { SPG49 (\# 615031) }\end{array}$ & TECPR2 & $\begin{array}{l}\text { Delayed psychomotor development, mental retardation } \\
\text { and spastic paraplegia (onset in the first decade), } \\
\text { dysmorphic features, thin corpus callosum }\end{array}$ & $\begin{array}{l}\text { An LC3 binding protein which associates with } \\
\text { trafficking proteins (e.g.SEC24D) and is required } \\
\text { ER export efficiency and in autophagosome } \\
\text { formation. }\end{array}$ & None reported \\
\hline $\begin{array}{l}\text { Autosomal recessive } \\
\text { pinocerebellar ataxia; } \\
\text { SCAR20 (\# 616354) }\end{array}$ & SNX14 & $\begin{array}{l}\text { Severely delayed psychomotor development and } \\
\text { intellectual disability, hypotonia, ataxia, absent speech, } \\
\text { relative macrocephaly, dysmorphic features }\end{array}$ & $\begin{array}{l}\text { Loss of SNX14 did not impact autophagosome- } \\
\text { lysosome fusion but results in increased } \\
\text { accumulation of autophagic organelles and } \\
\text { disruption of intracellular cholesterol } \\
\text { homeostasis. Functions in ER-lipid droplet } \\
\text { crosstalk and neutral lipid homeostasis between } \\
\text { these organelles }\end{array}$ & $\begin{array}{l}\text { Morpholino knockdown of snx14 in zebrafish } \\
\text { results in loss of neural tissue volume, } \\
\text { increased apoptosis and impaired autophagic } \\
\text { degradation [79] }\end{array}$ \\
\hline $\begin{array}{l}\text { Autosomal recessive } \\
\text { spastic paraplegia; } \\
\text { SPG11 (\# 604360) }\end{array}$ & SPG11 & $\begin{array}{l}\text { Hereditary spastic paraplegia, mental impairment, and } \\
\text { thin corpus callosum } \\
\text { Biallelic mutation in the SPG11 gene can also cause } \\
\text { autosomal recessive juvenile-onset amyotrophic lateral } \\
\text { sclerosis-5 (ALS5; 602099) and autosomal recessive } \\
\text { Charcot-Marie-Tooth disease type 2X (CMT2X; 616668), } \\
\text { different neurodegenerative disorders with overlapping } \\
\text { features }\end{array}$ & $\begin{array}{l}\text { SPG11 and SPG15 are essential for } \\
\text { autophagosome-lysosome reformation after } \\
\text { fusion and are required for lysosome biogenesis }\end{array}$ & $\begin{array}{l}\text { The Spg11 knockout mouse develop motor } \\
\text { impairment and cognitive deficits associated } \\
\text { with progressive brain atrophy with the loss } \\
\text { of neurons in the primary motor cortex, } \\
\text { cerebellum and hippocampus [80] }\end{array}$ \\
\hline $\begin{array}{l}\text { Autosomal recessive } \\
\text { spastic paraplegia 15; } \\
\text { SPG15\# (\# 270700) }\end{array}$ & $\begin{array}{l}\text { SPG15/ } \\
\text { ZFYVE26 }\end{array}$ & $\begin{array}{l}\text { Spastic paraplegia with other neurologic dysfunction, } \\
\text { including variable mental retardation, hearing and visual } \\
\text { defects, and thin corpus callosum }\end{array}$ & $\begin{array}{l}\text { SPG11 and SPG15 are essential for } \\
\text { autophagosome-lysosome reformation after } \\
\text { fusion and are required for lysosome biogenesis }\end{array}$ & $\begin{array}{l}\text { Zfyve26 knockout mice develop late-onset } \\
\text { spastic paraplegia with cerebellar ataxia [81] }\end{array}$ \\
\hline $\begin{array}{l}\text { Frontotemporal } \\
\text { dementia and/or } \\
\text { amyotrophic lateral } \\
\text { sclerosis 3; FTDALS3 } \\
\text { (\# 616437) }\end{array}$ & SQSTM1 & $\begin{array}{l}\text { Heterozygous mutations cause adult/late onset of } \\
\text { cognitive impairment, behavioural abnormalities, and } \\
\text { speech apraxia and/or upper and lower motor neuron } \\
\text { signs. Highly variable phenotype. Heterozygous mutation } \\
\text { in the SQSTM1 gene can also cause Paget disease of } \\
\text { bone and some patients may also develop this }\end{array}$ & $\begin{array}{l}\text { SQSTM1 is an autophagy adaptor protein that } \\
\text { can bind ubiquitinated substrates for selective } \\
\text { autophagy }\end{array}$ & $\begin{array}{l}\text { No neurological or bone phenotypes were } \\
\text { reported in sqstm1 knockout mice [82]. } \\
\text { These mice become obese as a result of } \\
\text { hyperphagia [83]. Phenotypes of } \\
\text { heterozygous mice were not reported }\end{array}$ \\
\hline $\begin{array}{l}\text { Infantile hypotonia } \\
\text { with psychomotor } \\
\text { retardation and } \\
\text { characteristic facies } \\
\text { 3; IHPRF3 (\# 616900) }\end{array}$ & TBCK & $\begin{array}{l}\text { Early onset neurodevelopmental disorder with poor } \\
\text { psychomotor development, poor speech, and inability to } \\
\text { walk independently }\end{array}$ & $\begin{array}{l}\text { Protein kinase that associates with the mitotic } \\
\text { apparatus and regulates cell size, cell } \\
\text { proliferation, and MTOR signalling. }\end{array}$ & None reported \\
\hline
\end{tabular}

Core/classical autophagy genes are shown in bold (although this definition is arbitrary to some extent). Defects in all of the genes affect autophagic flux. Main clinical

features taken from OMIM summaries (https://omim.org).

*Also known as beta-propeller protein-associated neurodegeneration; BPAN or static encephalopathy of childhood with neurodegeneration in adulthood; SENDA

\# also known as spastic paraplegia and retinal degeneration or Kjellin syndrome 


\section{Glossary Terms}

AMP-activated protein kinase (AMPK): AMPK phosphorylates and therefore regulates multiple components of the autophagy initiation pathway. It inhibits MTORC1 and activates ULK1.

ATG proteins: proteins encoded by the AuTophaGy-related (ATG) gene family.

Endosomal Sorting Complexes Required for Transport (ESCRT): Protein complexes involved in membrane remodelling during phagophore closure. When ESCRT machinery is disrupted, unclosed autophagosomes accumulate.

Lysosomal-Associated Membrane Protein 1 (LAMP1): a transmembrane protein found on lysosomes.

Macrocephaly: Macrocephaly is clinically described as an abnormally enlarged head. It may be caused by an enlarged brain or by accumulation of cerebrospinal fluid (hydrocephalus).

Microcephaly: A disorder where the brain fails to develop properly and can be identified by reduced head size. This may be evident at birth or within early childhood.

Mitophagy: The selective degradation of mitochondria by autophagy machinery.

Mammalian (or mechanistic) Target of Rapamycin (mTOR): A serine/threonine protein kinase that is a component of two different protein complexes, MTORC1 and MTORC2. In addition to regulating autophagy, MTORC1 regulates transcription and protein synthesis. MTORC2 is involved in the maintenance of the actin cytoskeleton and also activates insulin and insulin-like growth factor receptors. Rapamycin is a naturally occurring bacterial macrolide which inhibits MTOR and is commonly used experimentally to upregulate autophagy.

SNAREs: SNAp REceptor proteins are complexes involved in vesicle fusion.

ULK1: A kinase which phosphorylates several proteins required for the initiation of autophagy. ULK1 phosphorylates itself and can also be inhibited my MTORC1 and this reduces its activity. 


\section{Text boxes}

Box 1: ATG proteins

The core autophagic machinery and many of the regulatory complexes controlling autophagy initiation are encoded by a conserved family of approximately 30 genes, termed the AuTophaGy-related $(A T G)$ gene family. The $A T G$ genes were originally discovered in yeast where their mutation resulted in an inability to survive nutrient deprivation conditions. Hence these genes are functionally rather than structurally related. Many of the yeast genes have more than one vertebrate homolog, which may contribute to either redundancy or to additional functional diversity. For example, mammalian cells have six ATG8 orthologues; the MAP1LC3 (LC3) and GABARAP subfamilies (microtubule-associated protein 1 light chain 3 and $\mathrm{GABA}(\mathrm{A})$ receptor-associated protein families respectively).

Box 2: Non-canonical roles for ATG proteins

There is growing evidence that some autophagy proteins have functions in pathways that are independent from autophagy. Two examples of these are LC3-associated phagocytosis (LAP) and LC3-associated endocytosis (LANDO). In LAP, the canonical autophagy machinery is employed to conjugate LC3 to phagosomes, which have engulfed extracellular pathogens. Unlike in autophagy, LC3 lipidation occurs after the phagosome is sealed and it is proposed that the presence of LC3-II aids fusion with the lysosome [6]. LANDO describes the process whereby LC3 is conjugated to Rab5-positive, clathrin-positive endosomes and has been shown to function in microglia to regulate amyloid-beta clearance [5]. The roles of these processes may have widespread relevance to neuroimmunology. Autophagy machinery has also been described to play a role in the unconventional secretion pathways. The two inflammatory cytokines IL-1 $\beta$ and $1 \mathrm{~L}-18$, cytosolic proteins that lack conventional secretory signal sequences and therefore do not enter the ER-to-Golgi secretory pathway, have been shown to be excreted via autophagic machinery docking with the plasma membrane rather than being trafficked to the lysosome $[84,85]$. In addition, this may be a route for cytoplasmic organelles and large aggregates of proteins, common cargoes for conventional autophagy, to be extruded from cells. This route may also account for the egress and dissemination of intracellular microbes via autophagosome-like vesicles termed ejectosomes [86]. The autophagy proteins involved in LC3 lipidation have also recently been shown to play an important role in microtubule stability in an autophagy-independent fashion. The non-canonical role for these autophagy proteins is highly relevant in the context of neuronal plasticity [4]. 
Box 3: Using LC3 to measure autophagic flux

Quantifying autophagic flux is challenging, as there are no proteins (to our knowledge) that are degraded solely by autophagy and not by other additional routes. During autophagosome formation, ATG8-family proteins are conjugated to the lipid phosphatidylethanolamine (PE) in autophagosomal membranes. Since lipidated ATG8 proteins (such as LC3-II) are the only proteins which associate with pre-autophagosomal structures, autophagosomes and autolysosomes, they are widely accepted as being the best marker to distinguish autophagic vesicles from other cellular membranes. Measuring LC3 lipidation (LC3-II levels) by western blotting is one of the most common methods for measuring the number of autophagic vesicle and hence can be used to determine the rate of autophagic flux. Fluorescent or endogenous LC3 puncta can also be measured either by using fluorescently tagged reporters or antibody staining to recognise the endogenous protein, respectively. Unlipidated forms of LC3 often remains diffuse in the cytosol whereas LC3-II bound to vesicle membranes appear as bright puncta. However, increases in LC3-II or LC3 puncta may occur as a result of an increase in autophagosome formation (upregulation) or a blockage in clearance, therefore additional techniques are required to differentiate these two scenarios. For example, the use of lysosomal inhibitors clamps LC3-II degradation, and thus changes in LC3-II levels or LC3 vesicle numbers under such conditions can be inferred to be caused by altered autophagosome formation. 


\section{Figure Legends}

Figure 1: Autophagosome formation and degradation

A) The first morphologically recognizable autophagic precursors are called phagophores. These form within the cytoplasm as double-membraned, sac-like structures and can be recognised by the proteins that associate with their membranes, namely a complex of ATG12ATG5-ATG16L1 proteins and LC3-II. The edges of the phagophore elongate and fuse, and in doing so, engulf a portion of the cytoplasm. Just before the phagophore closes to form a vesicle, the ATG5-ATG12-ATG16L1 complex dissociates from the outer membrane, whereas LC3-II remains associated. The closed, double-membrane vesicle is called the autophagosome. Autophagosomes are trafficked along microtubules to the perinuclear region where they fuse with the lysosomes and their contents are degraded.

B) Lipidation of LC3-II: During autophagosome formation, LC3 (and other ATG8 family proteins) are conjugated to the lipid phosphatidylethanolamine (PE) in autophagosome membranes - this conjugated form is called LC3-II. This lipidation requires a protease and two ubiquitin-like conjugation systems (explained in [1]). ATG4 (a cysteine protease) cleaves the C-terminus of LC3 exposing a glycine residue. This first cleaved form of LC3 is called LC3I. A further reaction then occurs where ATG7 activates the C-terminal glycine residue. Next, the E2-like enzyme ATG3 and the ATG5-ATG12-ATG16L1 complex act together as an E3like ligase. This determines the site of LC3 lipidation and assists the transfer of LC3-I to PE in membranes to form LC3-II. The lipidated ATG8/LC3 proteins play a role in the expansion and closure of phagophore, in autophagosome-lysosome fusion and in degradation of the inner autolysosome membrane.

Figure 2: Overview of experimental evidence for the role of autophagy in neurogenesis and neuronal plasticity

Core autophagy genes are expressed in neuronal stem cells which give rise to neurons and astrocytes. Disruption of autophagy during developmental neurogenesis is associated with structural deficits; blocking autophagy in adult neural stem cells (NSCs) results in defects in adult neurogenesis and astrogenesis. Neuronal plasticity is the term used to describe the structural changes that occur within the brain throughout life, such as synaptic remodelling and dendritic pruning. Functional autophagy is required for these processes and blocking autophagy in vitro and in animal models results in reduced plasticity and consequently, problems with learning and memory. 


\section{Highlights}

- Growing evidence suggests that autophagy is essential for both developmental and adult neural stem cell maintenance, proliferation and differentiation.

- In the mature CNS, autophagy plays a role in plasticity through actions within the axon, dendritic spine, and during synaptic assembly.

- Defects in autophagy and its role in neurogenesis and neuronal plasticity may contribute to developmental disorders such as autism spectrum disorder and attention deficit hyperactivity disorder, memory deficits and psychiatric disorders such as depression. 


\section{Outstanding Questions Box}

- Since autophagy plays an essential role in neurogenesis, does altered autophagy in pregnancy have implications for lifelong health of the offspring?

- What are the functions of adult neural stem cells in CNS health and disease, and in view of autophagy's role in adult neural stem cell differentiation, how does autophagy contribute to these processes?

- To what extent does altered autophagy and its effects on neural plasticity contribute to psychiatric disease and neurobehavioural disorders? Is it possible to influence these by altering autophagy? 
Lysosome
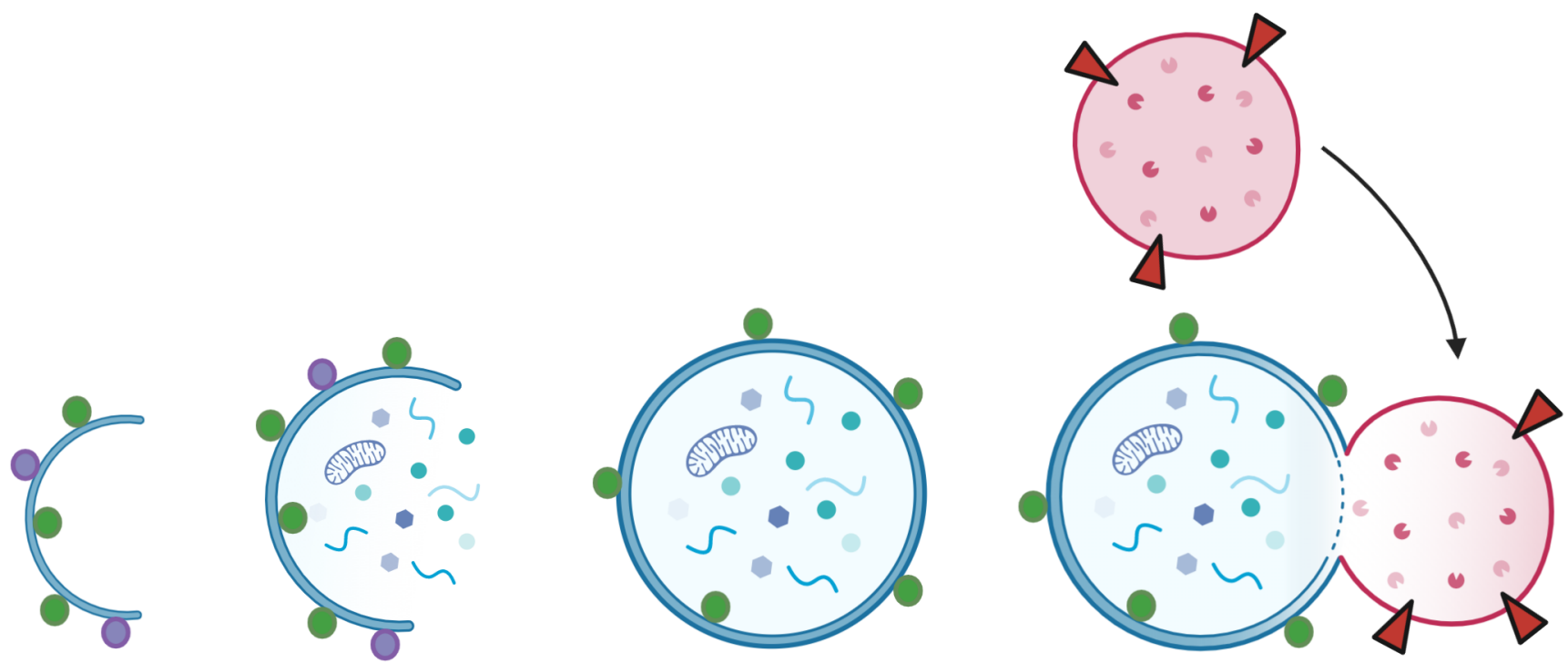

Phagophore

Autophagosome

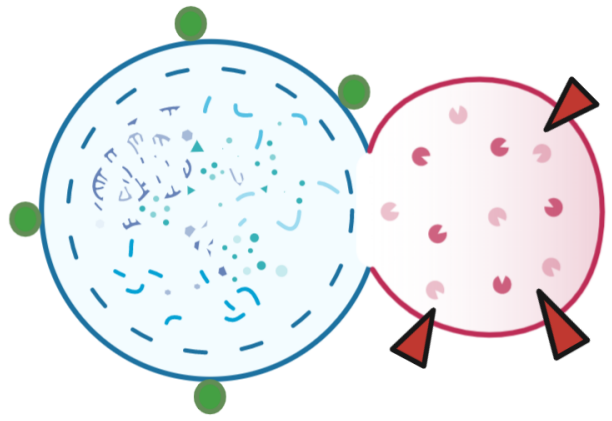

Autolysosome

- Atg5, Atg12, Atg16L1

- LC3-II

$\checkmark$ LAMP1

S. $\}$ Autophagic cargo

B

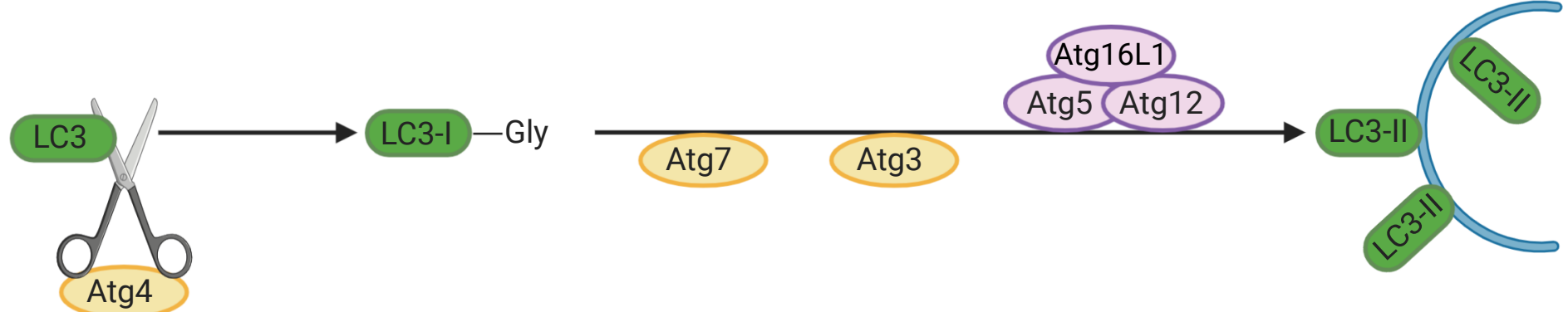

Figure One 
Stem cells produce neurons and astrocytes during development and during adult life

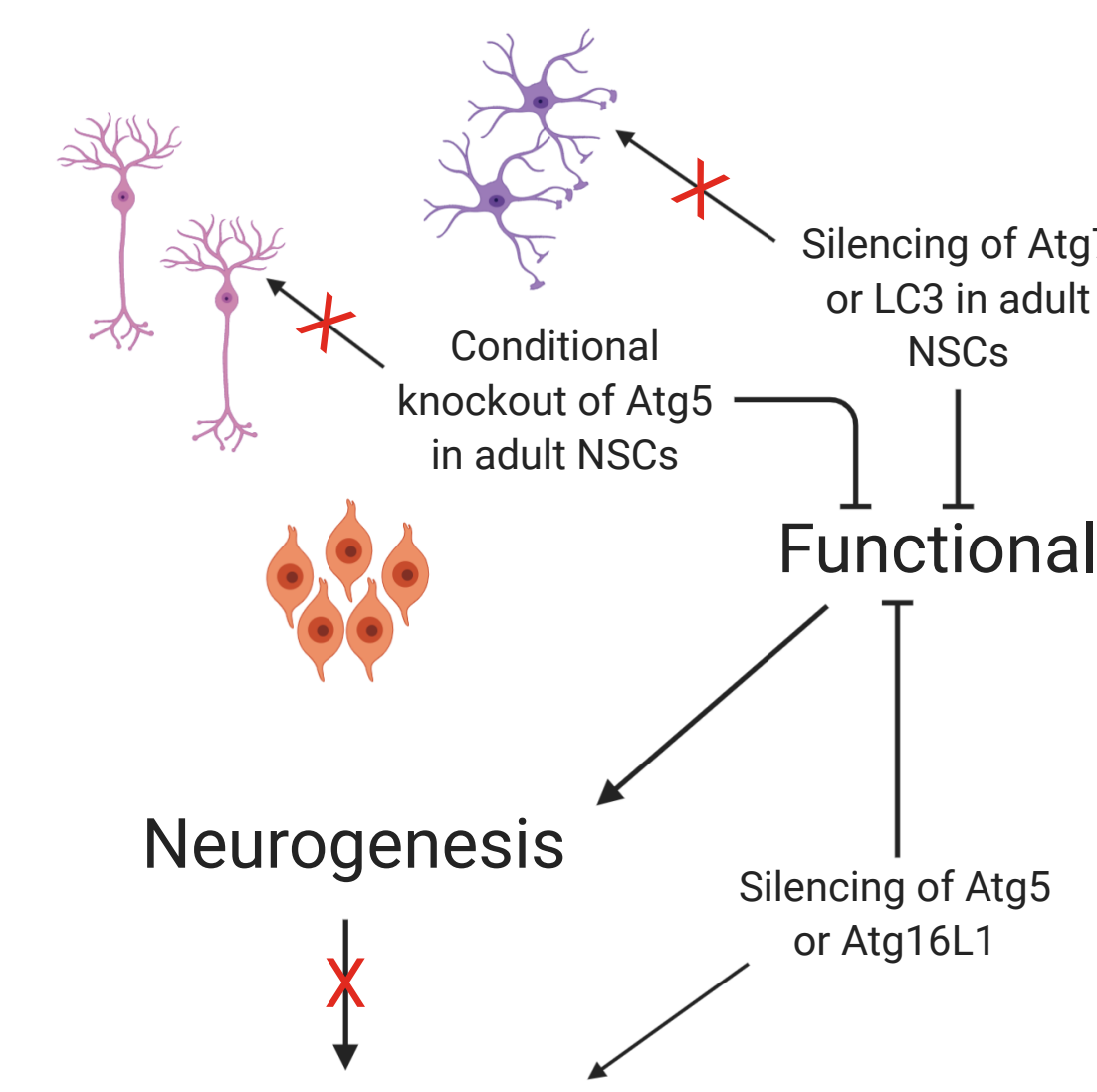

Deficits in neurogenesis result in reduced cortical thickness and structural deficits
Structural changes including dendrite pruning and synaptic turnover

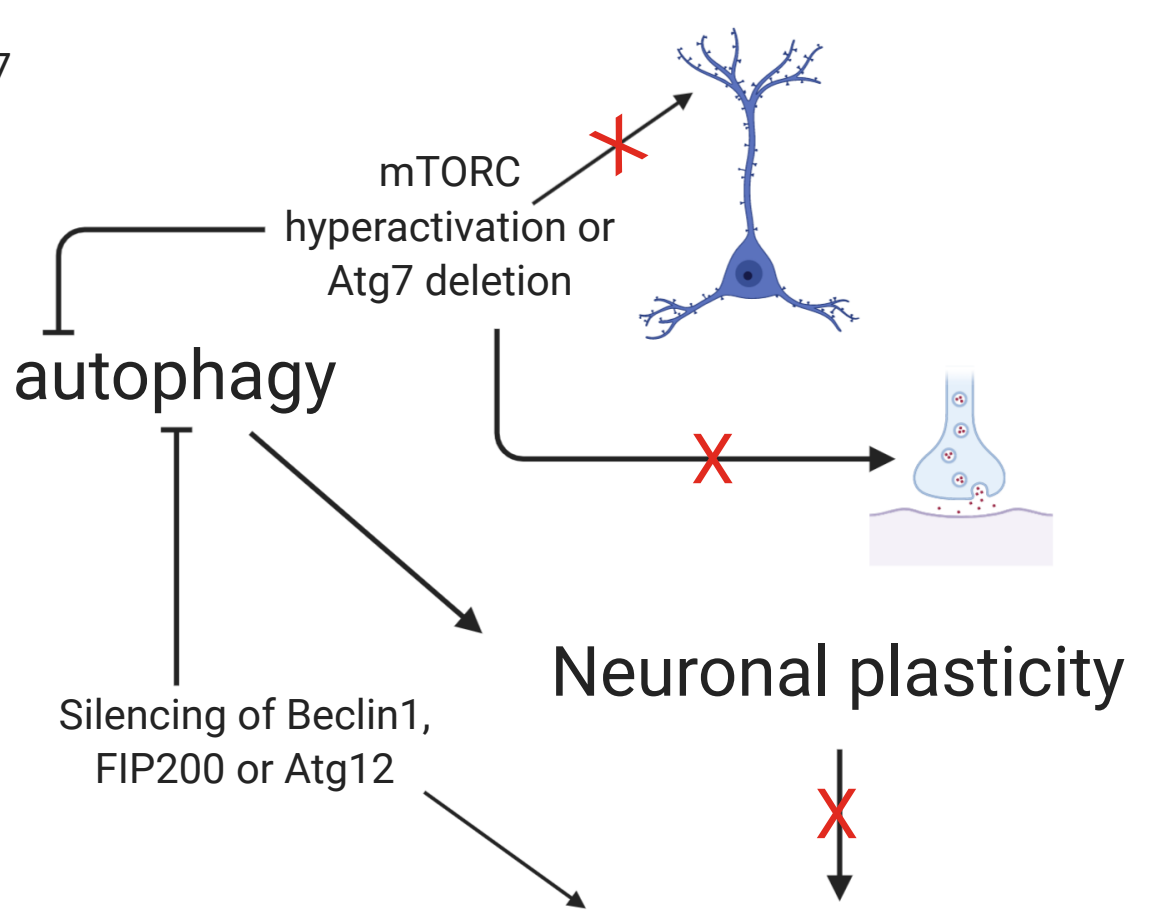

Defects in neuronal plasticity result in deficits in learning, memory and altered behaviours

Figure Two 